\title{
Lower gastrointestinal bleeding-Computed Tomographic Angiography, Colonoscopy or both?
}

\author{
Daniel Clerc ${ }^{1}$, Fabian Grass ${ }^{1}$, Markus Schäfer ${ }^{1}$, Alban Denys ${ }^{2}$, Nicolas Demartines ${ }^{1 *}$ and Martin Hübner ${ }^{1}$
}

\begin{abstract}
Background: Lower endoscopy (LE) is the standard diagnostic modality for lower gastrointestinal bleeding (LGIB). Conversely, computed tomographic angiography (CTA) offers an immediate non-invasive diagnosis visualizing the entire gastrointestinal tract. The aim of this study was to compare these 2 modalities with regards to diagnostic value and bleeding control.

Methods: Tertiary center retrospective analysis of consecutive patients admitted for LGIB between 2006 and 2012. Comparison of patients with LE vs. CTA as first exam, respectively, with emphasis on diagnostic accuracy and bleeding control.

Results: Final analysis included 183 patients; 122 (66.7\%) had LE first, while 32 (17.5\%) had CTA; 29 (15.8\%) had neither of both exams. Median time to CTA was shorter compared to LE (3 (IQR $=8.2)$ vs. $22(\mathrm{IQR}=36.9)$ hours, $P<0.001)$. Active bleeding was identified in 31\% with CTA vs. 15\% with LE ( $P=0.031)$; a non-actively bleeding source was found by CTA and LE in 22 vs. 31\%, respectively $(P=0.305)$. Bleeding control required endoscopy in 19\%, surgery in $14 \%$ and embolization in $1.6 \%$, while $66 \%$ were treated conservatively. Post-interventional bleeding was mostly controlled by endoscopic therapy (57\%). $80 \%$ of patients with active bleeding on CTA required surgery.

Conclusions: Post-interventional LGIB was effectively addressed by LE. For other causes of LGIB, CTA was efficient, and more available than colonoscopy. Treatment was conservative for most patients. In case of active bleeding, CTA could localize the bleeding source and predict the need for surgery.
\end{abstract}

Keywords: Gastrointestinal hemorrhage, Colonoscopy, Computed tomographic angiography, Endoscopy

\section{Background}

Lower gastrointestinal bleeding (LGIB) is a common clinical problem, representing 20 to $30 \%$ of patients presenting with gastrointestinal bleeding [1, 2]. LGIB incidence is increasing over time, as it is associated with older age and pre-existing comorbidities [3]. Very distal bleeding, e.g. due to hemorrhoids and low rectal cancer, is rather easy to diagnose, but bleeding from the colon and small bowel remains a diagnostic challenge. According to recent guidelines, hemodynamic stabilization and resuscitation must be performed prior to search any bleeding source. While

\footnotetext{
*Correspondence: demartines@chuv.ch

This study was presented at the Annual Swiss Surgical Meeting 2015, May

20-22, Bern, Switzerland

'Department of Visceral Surgery, University Hospital of Lausanne (CHUV),

Lausanne, Switzerland

Full list of author information is available at the end of the article
}

nasogastric lavage and/or esophagogastroduodenoscopy can be considered to rule out upper gastrointestinal bleeding for patients presenting severe hematochezia, lower endoscopy (LE) is the preferred diagnostic approach for LGIB $[1,4]$. Nevertheless, computed tomographic angiography (CTA) for evaluation of GI bleeding is increasingly used and may challenges lower endoscopy as most appropriate tool. This non-invasive diagnostic modality is readily available in most hospitals and can be rapidly performed without any bowel preparation. Reported sensitivity and specificity rates are 86 and $95 \%$, respectively and bleeding as low as $0.4 \mathrm{ml} / \mathrm{min}$ can be detected $[5,6]$.

So far, there is only limited evidence on the routine use of CTA in the initial management of LGIB. Its accuracy compared to LE remains unclear; and subsequently, its 
use as a first-line diagnostic modality is not considered in current management algorithms $[1,4]$.

This current study aimed to compare the accuracy of CTA and colonoscopy in the diagnosis of LGIB and their influence on bleeding control.

\section{Methods}

Retrospective cohort study conducted at a tertiary care academic center. The study was approved by the local ethics committee (protocol 389/12) performed according the STROBE recommendations, and registered under www.researchregistry.com (\# 726).

\section{Patients and data collection}

All consecutive patients admitted for LGIB to the department of visceral surgery between January 2006 and December 2012 were potentially eligible. The following patients were excluded from analysis: (I) proctological bleeding, (II) LGIB not being the primary cause of admission, and (III) patients with LGIB admitted for elective surgery. Patients underwent primary evaluation at the emergency department by emergency physicians and by a gastrointestinal consultant surgeon before admission to the visceral surgery department. The initial diagnostic modality (LE or CTA) was defined by the physician in charge of the patient.

LE was defined as a flexible lower endoscopy performed by a gastroenterologist. Procedures were performed by an attending, junior staff or resident gastroenterologist according to the policy of a Swiss university hospital. Resident procedures were supervised by a board-certified gastroenterologist. Gastroenterology consultant is available 24/7 in our institution, with in-hospital presence during daytime, and available within 30 min during the nightshift. Type of bowel preparation, if any, was upon the choice of the gastroenterologist performing the procedure. A standard flexible colonoscope was used for all procedures. Conscious sedation or general anesthesia was used depending upon patient's general condition. Whenever possible, examination was performed up to the ileo-caecal valve. Exam findings were classified as positive with actively bleeding lesion, positive with non-actively bleeding lesion or inconclusive. LE was defined inconclusive when no lesion was detected regardless of the quality of preparation and the amount of blood clots present in the endoscopic view.

For actively bleeding lesions or non-actively bleeding lesions with high risk of re-bleeding, endoscopic therapy was directly applied with clips, adrenaline infusion or thermal probe depending on the gastroenterologist's preference.

CTA was defined as a contrast-enhanced abdominal and pelvic $\mathrm{CT}$ scan performed in a triphasic acquisition. First, a native acquisition was followed by contrastenhanced arterial phase after a bolus injection of $100 \mathrm{ml}$ contrast media (300mg iodine $/ \mathrm{ml}, 4 \mathrm{ml} / \mathrm{s}$ ) with automatic triggering (collimation $16 \times 0.625 \mathrm{~mm}$; pitch 1.75 ; table speed $35 \mathrm{~mm} / \mathrm{s}$ ). The venous acquisition was performed 70-80 s later. Exam findings were classified as positive with active bleeding when CTA showed intraluminal contrast material extravasation. CTA were defined positive without active bleeding when the cause of bleeding was spotted without contrast material extravasation. CTA were defined inconclusive when no cause of bleeding was found.

The time spent from the admission to the emergency room to the execution of the diagnostic exams was recorded. First hemodynamic parameters (heart rate (HR), blood pressure) and hemoglobin $(\mathrm{Hb})$ values recorded upon admission were retrieved. The shock index (SI), defined as the ratio of heart rate to systolic blood pressure, and the mean arterial pressure (MAP), were calculated.

The cause of bleeding was classified in six categories: small bowel, diverticular, colorectal neoplasia, colorectal lesion, post-interventional, and unknown location. Small bowel bleeding was defined as the source of bleeding arising from the ligament of Treitz to the ileo-caecal valve. Diverticular bleeding was defined as the source of bleeding being related to a colonic diverticular disease. A colorectal neoplasia was defined as benign or malignant lesion being the cause of bleeding. Non-neoplastic, non-diverticular colorectal lesions being the source of bleeding (colitis, colonic ulcers or angiodysplasias) were grouped in the colorectal lesion category. Post-interventional bleeding was defined as a bleeding occurring following endoscopic or surgical procedure. Bleeding of unknown location was defined when the cause of bleeding was not found during hospital stay. The control of bleeding was recorded according to the last therapeutic intervention performed (surgery, angio-embolization, endoscopic intervention or conservative).

Patient's characteristics, information on the performed diagnostic procedures and clinical outcomes were defined a priori. All eligible patients were collected by ICD codes. Data was retrieved by retrospective chart review and entered in a coded computerized database. Patients were stratified according to the first diagnostic exam performed at hospital admission, i.e. LE, CTA or none of both exams.

\section{Statistical analysis}

Descriptive statistics for categorical variables were reported as frequency (\%), while continuous variables were reported as median (interquartile range). Chi-square and Student's $t$-test were used for comparison of categorical and continuous variables, respectively. All statistical tests were two-sided and a level of 0.05 was used to indicate statistical significance. Data analysis was performed with the Statistical Software for the Social Sciences SPSS 
Advanced Statistics 22 (IBM Software Group, 200 W. Madison St., Chicago, IL; 60606 USA).

\section{Results}

\section{Patients and clinical outcome}

Within the study period, 301 patients were hospitalized in the visceral surgery department with diagnostic of GIB. 118 patients were excluded according to the $a$ priori defined rules. Final analysis included 183 patients, with 109 male and 74 female patients with a median age of 75 years (Fig. 1). One hundred and twenty-two patients $(66.7 \%)$ had LE as first diagnostic intervention, 32 (17.5\%) had CTA. The remaining 29 patients (15.8\%) had neither of the two exams during their hospital stay. Patient characteristics are presented in Table 1. Median length of hospital stay (LOS) was $5(\mathrm{IQR}=7)$ days. In the CTA group, LOS was longer compared to the LE group with $9(\mathrm{IQR}=12.7)$ versus $5(\mathrm{IQR}=6.6)$ days, respectively $(P=0.026)$. In-hospital mortality rate was $2.7 \%$ and concerned 5 patients. 3 patients died of postoperative complications (septic shock, intravascular disseminated coagulopathy, cardiac failure), one patient of multi-organ failure following hemorrhagic shock and one patient presented sudden cardiac arrest. Four out of these 5 deceased patients were examined with CTA first. Twenty-eight patients $(15.3 \%)$ were referred after initial evaluation in a regional center. The CTA group had the higher proportion of referred patients (34.4\%) compared with the LE group (9.8\%). Patients' hemodynamics are detailed in
Table 1 Patient characteristics

\begin{tabular}{lllll}
\hline & $\begin{array}{l}\text { All patients } \\
(n=183)\end{array}$ & $\begin{array}{l}\text { CTA } \\
(n=32)\end{array}$ & $\begin{array}{l}\text { LE } \\
(n=122)\end{array}$ & $P$ \\
\hline Age, median (range) & $75(21-99)$ & $68(21-94)$ & $77(33-99)$ & $\mathbf{0 . 0 0 4}$ \\
Male, n (\%) & $109(59.6)$ & $19(59.4)$ & $71(58.2)$ & 0.909 \\
Hb (g/L), Mean \pm SD & $110 \pm 27$ & $102 \pm 30$ & $112 \pm 24$ & 0.090 \\
MAP (mmHg), Mean \pm SD & $93 \pm 18$ & $85 \pm 18$ & $95 \pm 16$ & $\mathbf{0 . 0 0 6}$ \\
HR, Mean \pm SD & $83 \pm 16$ & $82 \pm 15$ & $83 \pm 16$ & 0.881 \\
SI, Mean \pm SD & $0.66 \pm 0.22$ & $0.72 \pm 0.27$ & $0.63 \pm 0.18$ & 0.082 \\
Diagnostic, $n$ (\%) & & & & \\
Diverticular & $48(26.2)$ & $9(28.1)$ & $38(31.1)$ & 0.741 \\
Unknown location & $41(22.4)$ & $5(15.6)$ & $21(17.2)$ & 0.831 \\
Colo-rectal lesion & $34(18.6)$ & $5(15.6)$ & $27(22.1)$ & 0.419 \\
Post-interventional & $23(12.6)$ & $2(6.2)$ & $17(13.9)$ & 0.239 \\
Small bowel & $20(10.9)$ & $11(34.4)$ & $4(3.3)$ & $<0.001$ \\
Colo-rectal neoplasia & $17(9.3)$ & $0(0)$ & $15(12.3)$ & $\mathbf{0 . 0 3 7}$
\end{tabular}

Comparison of baseline characteristics of patients who had CTA and patients who had LE. Significant $P$-values $(<0.05)$ are indicated in bold characters. $S \mathrm{I}$ is defined as HR/Systolic blood pressure

CTA Computed tomographic angiography, LE Lower endoscopy, Hb Hemoglobin level, MAP Mean arterial pressure, HR Heart rate, SI Shock index

Table 1. MAP was significantly lower in the CTA group, compared with the LE group with $85 \pm 18$ versus $95 \pm 16$ $\mathrm{mmHg}$, respectively $(P=0.006)$. Patients in the CTA group had similar Hb levels at admission compared with the LE group $(102 \pm 30$ versus $112 \pm 24 \mathrm{~g} / \mathrm{L}$, respectively; $P=0.09$ ). SI was also similar in patients in the CTA group,

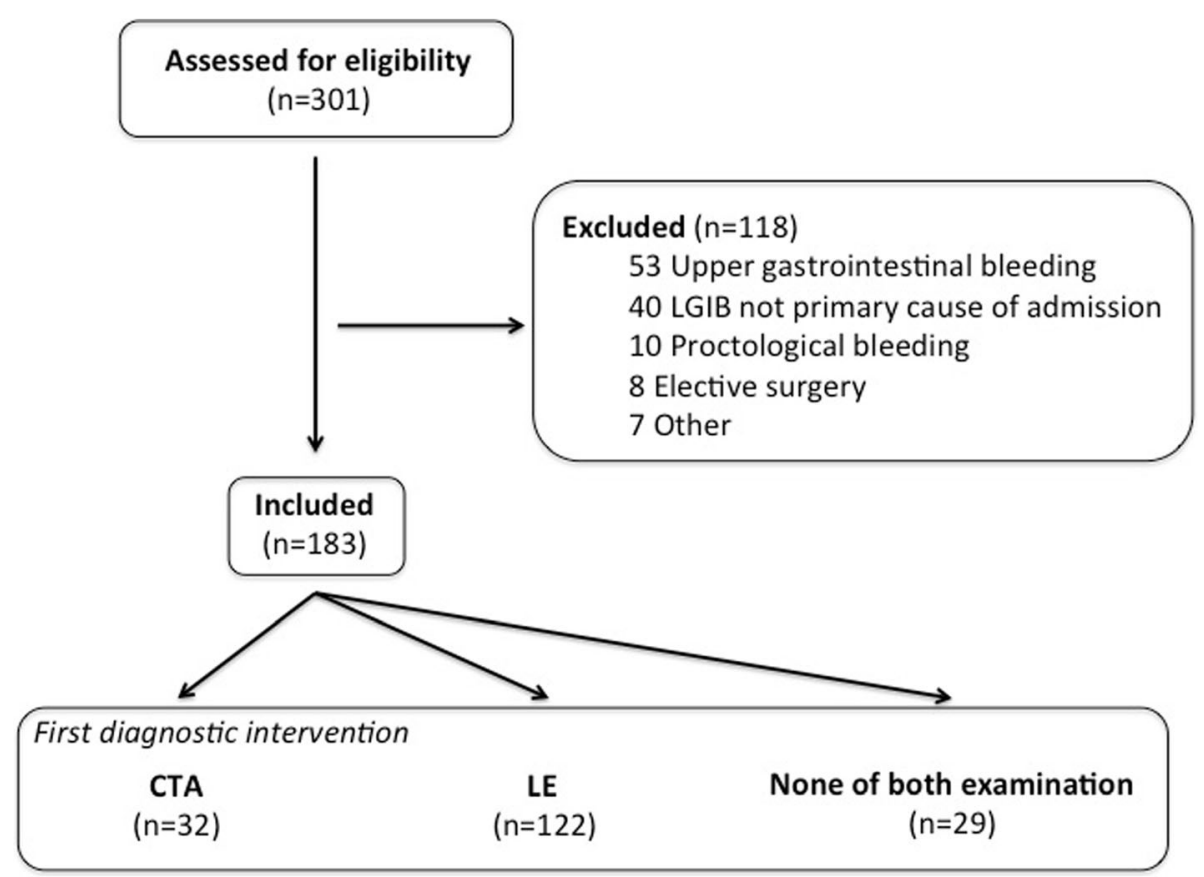

Fig. 1 Study flow chart. Lower gastrointestinal bleeding (LGIB), Computed tomographic angiography (CTA), Lower endoscopy (LE) 
with $0.72 \pm 0.27$ versus $0.63 \pm 0.18$ in those of LE group $(P=0.082)$. No significant difference was observed in the comparison of $\mathrm{HR}$ at admission in both groups (Table 1).

\section{Radiological and endoscopic findings}

In the first 3 years of the study period, LE was the predominating diagnostic tool, while CTA gained of importance in the second part of the study period only (Fig. 2).

Following hospital admission, CTA was performed significantly earlier than colonoscopy, after a median of 3 $(\mathrm{IQR}=8.2)$ versus $22(\mathrm{IQR}=36.9)$ hours $(P<0.001)$.

Active bleeding was found significantly more frequently with CTA compared to LE (31.3 vs. $14.8 \%, P=0.031)$. A non-active bleeding source was identified in $21.8 \mathrm{vs}$. $31.1 \%$ by CTA and LE, respectively $(P=0.305)$. The rate of inconclusive exams was similar in both groups $(46,9$ vs. $54.1 \%, P=0.396)$.

Patients presenting post-interventional bleeding were mostly evaluated by LE first, and rarely by CTA first (73.9 vs. $8.7 \%$, respectively). Patients with small bowel bleeding underwent predominantly CTA first (55\%) compared to LE first (20\%), this difference was statistically significant $(P<0.001)$.

\section{Bleeding control}

The control of bleeding was achieved by conservative measures in $120(65.6 \%)$ patients, by endoscopic intervention in $34(18.6 \%)$, by surgery in $26(14.2 \%)$ and by embolization in 3 patients (1.6\%), respectively. There were no differences in the rate of conservatively managed patients in the CTA group compared to the LE group ( 56.3 vs. $61.5 \%, P=0.591$ ). A summary of the final bleeding control according to the first exam used is shown in Fig. 3. Active bleeding on CTA was found in 10 patients and surgery was needed for the final control of the bleeding in $80 \%$ of cases, whereas the remaining $20 \%$ of patients were treated conservatively. After positive CTA for these 10 patients, 4 underwent surgery directly, 3 LE and 3 angiography. All 3 LE were inconclusive because of impaired vision due to blood clots, and only 1 out of 3 angiographies was positive allowing embolization in one single patient who eventually needed surgery for re-bleeding. In all these cases except for one, the bleeding localization was confirmed by the pathology report. Patients presenting post-interventional bleeding were primarily controlled by LE in $57 \%$. Patients with small bowel bleeding needed more often surgery (35\%) compared with other pathologies (Fig. 4). Of the 29 patients with no exam during hospital stay, 27 patients had conservative treatment and 2 underwent immediate invasive treatment due to hemodynamic instability. Embolization was performed for 1 patient and 1 patient underwent surgery directly. The remaining 27 patients were not further examined because of clinically minor bleeding, with spontaneous resolution.

\section{Discussion}

The results of this study suggest that CTA may be a good choice in most patients with LGIB despite the fact that LE was the most used modality in our series and remains best choice for post-interventional bleeding. CTA was quickly available, reliable to indentify the bleeding source and helpful to guide further management.

In the group of patients assessed by CTA first, a significantly greater rate of active bleeding was observed compared to patients examined with LE first (31.3 vs. $14.8 \%$, respectively, $P=0.031$ ). While the shorter waiting

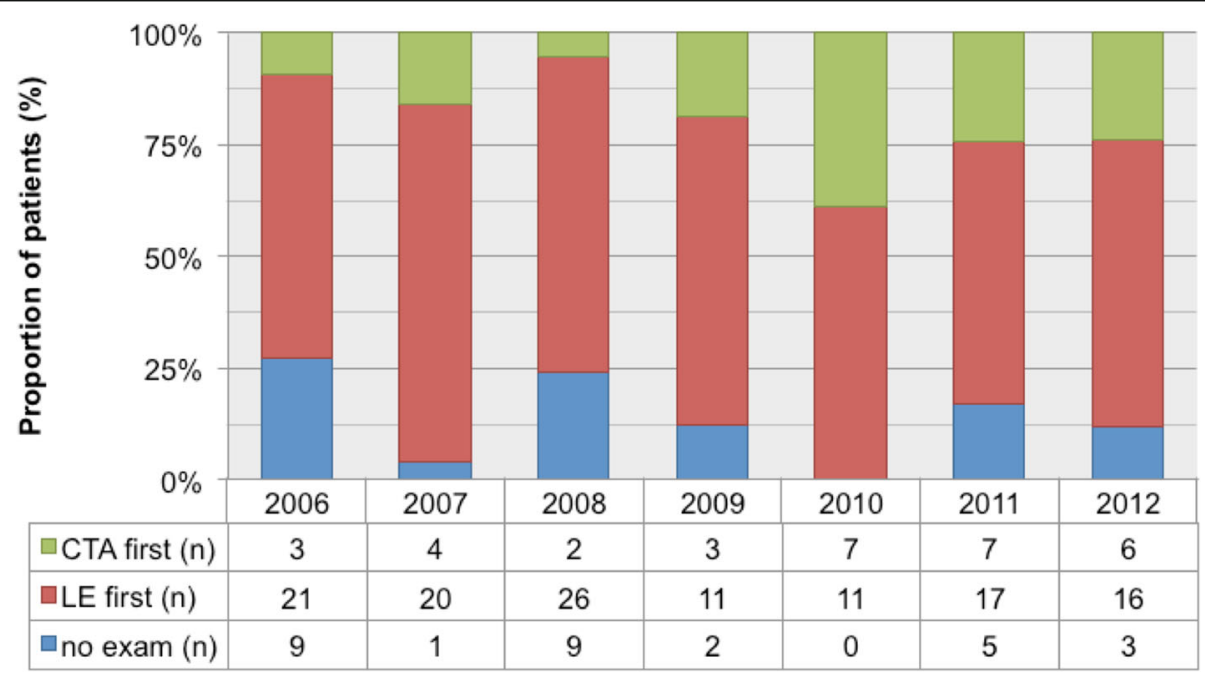

Fig. 2 Proportion of first diagnostic intervention per year. Computed tomographic angiography (CTA), Lower endoscopy (LE) 


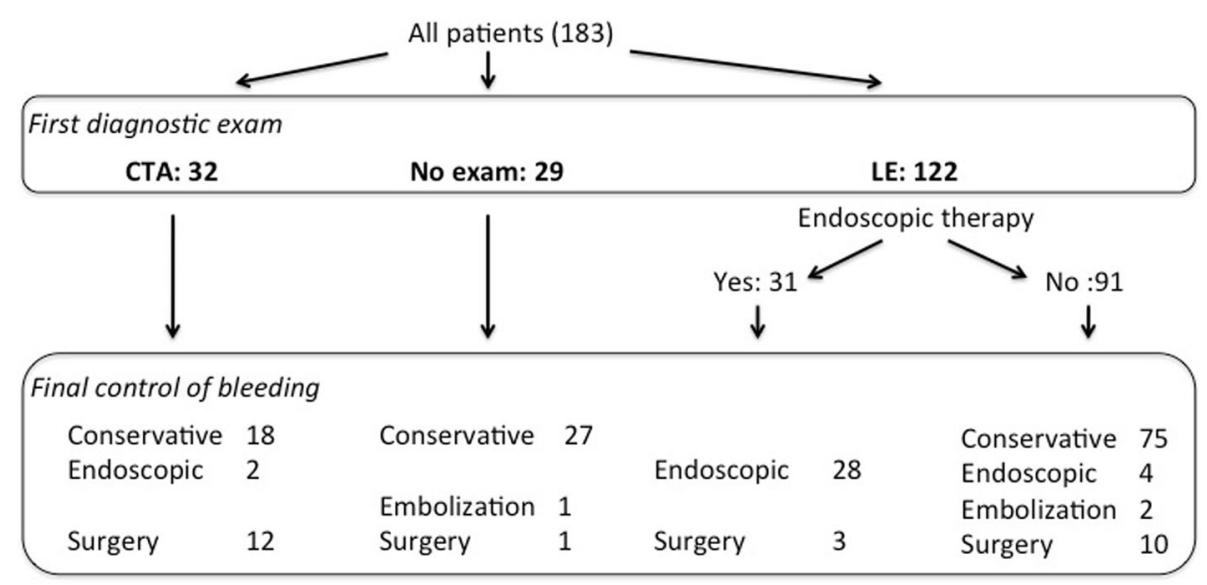

Fig. 3 Flow chart of the bleeding control according to the first exam used. Values are presented as number of patients. Computed tomographic angiography (CTA), Lower endoscopy (LE)

time in the CTA group very likely increased the chance to identify actively bleeding lesions, the delay taken to perform LE increased the chance for spontaneous bleeding cessation. Delay in performing LE is explained by prior bowel preparation and limited gastroenterology consultant availability out of office hours. Nonetheless, about half of the exams were inconclusive in both groups, without significant difference. In a study including 115 patients with LGIB who underwent CTA, Chan \& al. found that $77 \%$ of patients with negative studies did not need further intervention. In $68 \%$ of cases the exam did not show features of active bleeding, which is consistent with the present findings [7].

In the literature, the optimal time point of colonoscopy for LGIB remains controversial. In a retrospective analysis, Strate \& al. revealed that shorter time to colonoscopy was an independent predictor of shorter LOS, particularly if colonoscopies were performed within 12-24 h [8]. A further trial confirmed that the source of bleeding was more frequently found with urgent colonoscopy (within $8 \mathrm{~h}$ ) compared to elective colonoscopy (within 4 days) [9]. On the other hand, in a randomized trial comparing colonoscopies performed within $12 \mathrm{~h}$ of presentation to those executed within 36-60 h, the authors did not show differences in clinical outcomes. However non-diagnostic colonoscopies were more common in the elective group [10].

In this series, $65.6 \%$ of patients had spontaneous cessation of bleeding regardless of the first diagnostic exam chosen. This suggests that most patients presenting with LGIB can be managed conservatively. In the literature, self-limiting LGIB rates of up to $80 \%$ have been reported, especially in case of diverticular bleeding $[4,11,12]$.

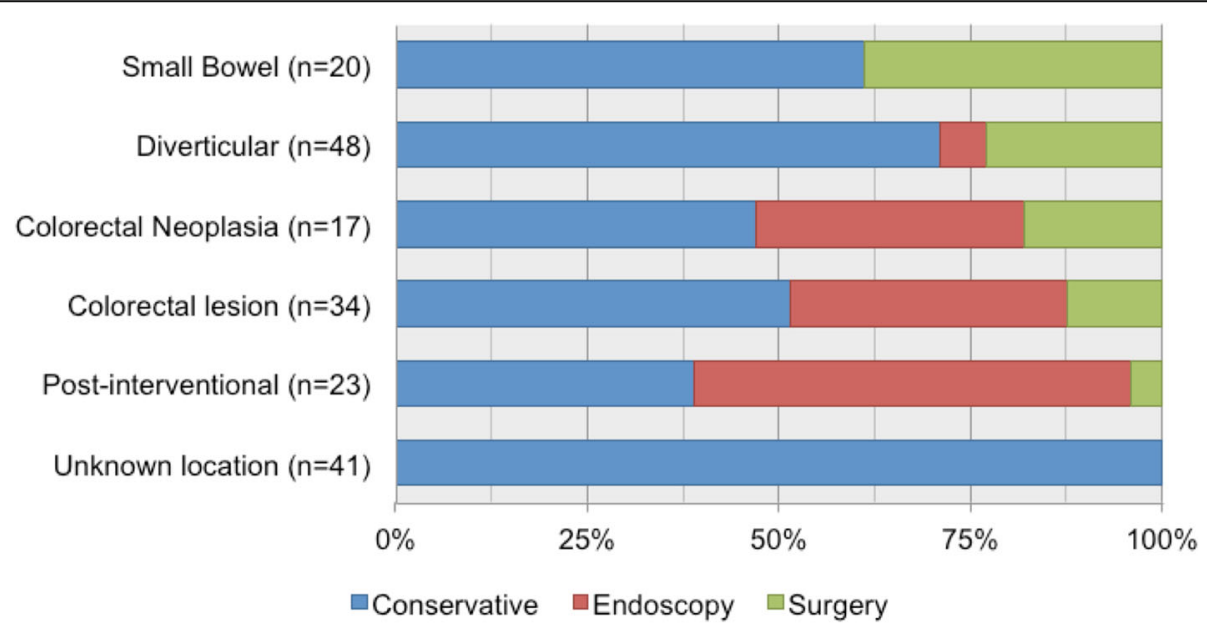

Fig. 4 Bleeding control according to the final diagnostic 
Surgery was required in $14 \%$ of patients in this serie which is similar to the published range of 2.6 to $18 \%$ $[8,9,13,14]$. In-hospital mortality was $2.7 \%$ in the present study. Previously published mortality rates ranged from 2.4 to $8.8 \%$, $[3,8,13,15]$.

The present results suggest that most patients with post-interventional bleeding were effectively treated with colonoscopy. In this sub-group of patients, examination with CTA was not necessary, and patients should directly undergo early colonoscopy to confirm diagnosis and deliver treatment at the same time. In the literature, several authors reported successful endoscopic management of post-polypectomy bleeding for most patients [16-18]. In the present series, $80 \%$ of patients with active bleeding on CTA required surgery for bleeding control. This is in line with Chan \& al. reporting about $90 \%$ of patients with LGIB and positive CTA needing intervention for bleeding control. However, surgery was performed in $24 \%$ of cases whereas embolization was successful in $64 \%$ of patients [7]. In a study from Nagata \& al., early colonoscopy following CTA resulted in a higher detection rate of colonic vascular lesions than colonoscopy alone [19]. These results contrast the present findings were all $3 \mathrm{LE}$ performed after positive CTA were negative due to impaired visualization. Koh \& al. suggested that angiography should be performed as soon as possible after positive CTA to allow embolization [20]. In their series, angiography was performed only after CTA with signs of active bleeding and about half of them were negative. In the present study, only 3 patients underwent angiography after positive CTA, 2 of them were negative and one patient underwent embolization first, followed by salvage surgery because of early re-bleeding.

Based on these findings, our own institutional algorithm for the management of LGIB was adapted (Fig. 5). For post-interventional bleeding, urgent LE is advocated.
Patients presenting with minor bleeding can be observed and prepared for elective colonoscopy within $24 \mathrm{~h}$. Patients presenting with more significant bleeding should undergo CTA as first-line procedure. It is hypothesized that this algorithm may help to decrease time to diagnosis and guide successful treatment. In published practice guidelines algorithms, radionuclide red blood cell scan followed by mesenteric angiography is indicated for patients unfit for colonoscopy or those with failed endoscopic therapy, but use of CTA is not included $[1,4]$. Other authors have integrated CTA in their management algorithm. Copland \& al. proposed, for clinically active bleeding, CTA as first-line procedure after exclusion of UGIB with nasogastric lavage. Colonoscopy is then performed if CTA localized the bleeding [21]. Another report proposed an algorithm including CTA for all patients with LGIB [22]. Chan \& al. proposed a management pathway including CTA after negative endoscopic evaluation [7]. The fact that so much various algorithms are proposed suggests that further research is needed.

This study has several limitations besides its retrospective design and a limited number of patients. Within the study period, there was a change in care providers and hence in practice. Furthermore, the choice of the first diagnostic exam was decided by the treating physician. Significant lower MAP and trends to lower $\mathrm{Hb}$ level and higher SI indicate a selection bias as the severely affected patients were more likely to undergo CTA first. Longer LOS in the CTA group also probably reflects the greater severity of the bleeding. Patients' comorbidites, medication, medical or surgical history also probably led to a selection bias in the choice of the first exam. The retrospective nature of the study precluded us to ensure a valid comparison. We could not account for the delay in performing LE during the night, as the gastroenterology consultant's availability was lower outside office hours.

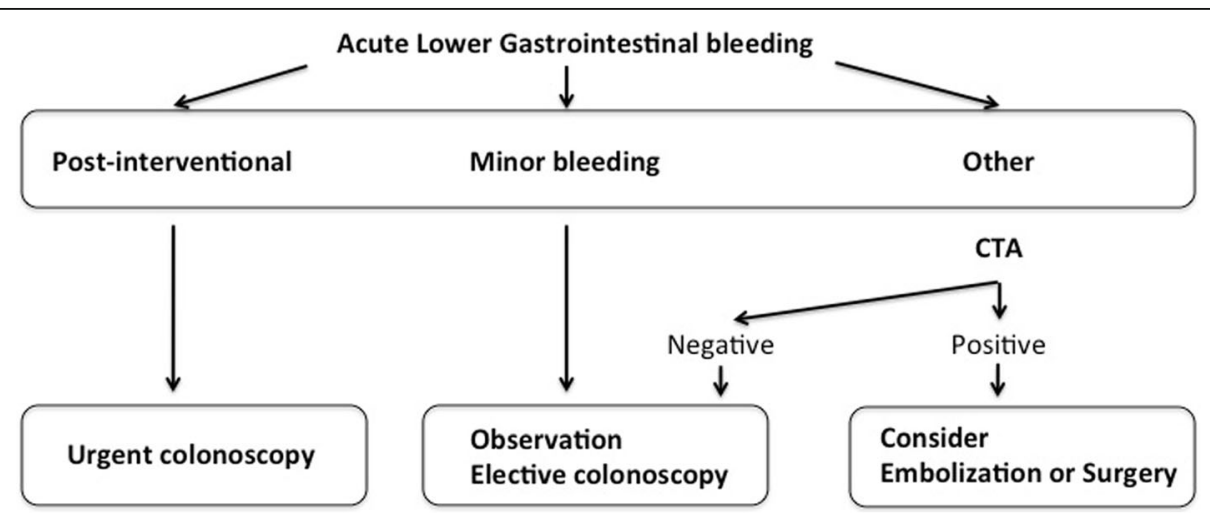

Fig. 5 Management of acute lower gastrointestinal bleeding, proposed algorithm. Minor bleeding is defined as patients presenting without signs of hemodynamic comprise, need for transfusion or ongoing bleeding. Computed tomographic angiography (CTA) 


\section{Conclusion}

In conclusion, our analysis describes current practice of management for LGIB in a "real-world" setting in order to propose a new treatment algorithm that needs to be evaluated prospectively. The present study suggests that CTA is an efficient and readily available tool to manage patients with LGIB. CTA could be considered a suitable first diagnostic option for acute LGIB except for postinterventional bleeding which should entail immediate LE. However, most patients with LGIB can be treated conservatively.

\section{Acknowledgements}

Not applicable.

\section{Funding}

Not applicable.

\section{Availability of data and material}

The data supporting the findings of this study are available from University Hospital of Lausanne (CHUV) but restrictions apply to the availability of these data, which were used under permission for the current study, and so are not publicly available. Data are however available from the authors upon reasonable request and with permission of University Hospital of Lausanne (CHUV).

\section{Authors' contributions}

DC: design, analysis and interpretation, drafting/FG: analysis and interpretation, critical revision/MS: design, interpretation, critical revision/AD: design, interpretation, critical revision/ND: conception, interpretation, critical revision/ $\mathrm{MH}$ : conception and design, analysis and interpretation, drafting/All authors approved the final version.

\section{Competing interests}

The authors declare that they have no competing interests.

\section{Consent for publication}

Not applicable.

\section{Ethics approval and consent to participate}

The study was approved by the ethics committee of the University of Lausanne (protocol 389/12).

\section{Author details}

'Department of Visceral Surgery, University Hospital of Lausanne (CHUV), Lausanne, Switzerland. ${ }^{2}$ Department of Interventional Radiology, University Hospital of Lausanne (CHUV), Lausanne, Switzerland.

Received: 4 November 2016 Accepted: 14 December 2016

Published online: 03 January 2017

\section{References}

1. ASGE Standards of Practice Committee, Pasha SF, Shergill A, Acosta RD, Chandrasekhara V, Chathadi KV, Early D, Evans JA, Fisher D, Fonkalsrud L, Hwang JH, Khashab MA, Lightdale JR, Muthusamy VR, Saltzman JR, Cash BD. The role of endoscopy in the patient with lower Gl bleeding. Gastrointest Endosc. 2014;79(6):875-85. doi:10.1016/j.gie.2013.10.039. Epub 2014 Apr 2.

2. Strate LL. Lower Gl, bleeding: epidemiology and diagnosis. Gastroenterol Clin North Am. 2005:34(4):643-64. Review.

3. Lanas A, García-Rodríguez LA, Polo-Tomás M, Ponce M, Alonso-Abreu L, Perez-Aisa MA, Perez-Gisbert J, Bujanda L, Castro M, Muñoz M, Rodrigo L, Calvet $X$, Del-Pino D, Garcia S. Time trends and impact of upper and lower gastrointestinal bleeding and perforation in clinical practice. Am J Gastroenterol. 2009;104(7):1633-41. doi:10.1038/ajg.2009.164. Epub 2009 May 5.

4. Zuccaro Jr G. Management of the adult patient with acute lower gastrointestinal bleeding. American College of Gastroenterology. Practice Parameters Committee. Am J Gastroenterol. 1998;93(8):1202-8.
5. Chua $A E$, Ridley LJ. Diagnostic accuracy of $C T$ angiography in acute gastrointestinal bleeding. J Med Imaging Radiat Oncol. 2008;52(4):333-8. doi:10.1111/j.1440-1673.2008.01964.x

6. Geffroy Y, Rodallec MH, Boulay-Coletta I, Jullès MC, Ridereau-Zins C, Zins M. Multidetector CT angiography in acute gastrointestinal bleeding: why, when, and how. Radiographics. 2011;31(3):E35-46. Review. Erratum in: Radiographics. 2011 Sep-Oct;31(5):1496. Radiographics. 2011 Nov-Dec;31(7): 2114. Fullès, Marie-Christine [corrected to Jullès, Marie-Christine].

7. Chan V, Tse D, Dixon S, Shrivastava V, Bratby M, Anthony S, Patel R, Tapping C, Uberoi R. Outcome following a negative CT Angiogram for gastrointestinal hemorrhage. Cardiovasc Intervent Radiol. 2015;38(2):329-35. doi:10.1007/s00270-014-0928-8. Epub 2014 Jul 15.

8. Strate LL, Syngal S. Timing of colonoscopy: impact on length of hospital stay in patients with acute lower intestinal bleeding. Am J Gastroenterol. 2003:98(2):317-22

9. Green BT, Rockey DC, Portwood G, Tarnasky PR, Guarisco S, Branch MS, Leung J, Jowell P. Urgent colonoscopy for evaluation and management of acute lower gastrointestinal hemorrhage: a randomized controlled trial. Am J Gastroenterol. 2005;100(11):2395-402.

10. Laine $L$, Shah A. Randomized trial of urgent vs. elective colonoscopy in patients hospitalized with lower Gl bleeding. Am J Gastroenterol. 2010; 105(12):2636-41. doi:10.1038/ajg.2010.277. quiz 2642. Epub 2010 Jul 20.

11. Barnert J, Messmann H. Diagnosis and management of lower gastrointestinal bleeding. Nat Rev Gastroenterol Hepatol. 2009;6(11):637-46. doi:10.1038/nrgastro.2009.167. Review.

12. Farrell JJ, Friedman LS. Review article: the management of lower gastrointestinal bleeding. Aliment Pharmacol Ther. 2005;21(11):1281-98. Review.

13. Strate LL, Saltzman JR, Ookubo R, Mutinga ML, Syngal S. Validation of a clinical prediction rule for severe acute lower intestinal bleeding. Am J Gastroenterol. 2005;100(8):1821-7.

14. Jacovides CL, Nadolski G, Allen SR, Martin ND, Holena DN, Reilly PM Trerotola S, Braslow BM, Kaplan LJ, Pascual JL. Arteriography for lower gastrointestinal hemorrhage: role of preceding abdominal computed tomographic angiogram in diagnosis and localization. JAMA Surg. 2015; 150(7):650-6. doi:10.1001/jamasurg.2015.97.

15. Strate LL, Ayanian JZ, Kotler G, Syngal S. Risk factors for mortality in lower intestinal bleeding. Clin Gastroenterol Hepatol. 2008 Sep;6(9):1004-10; quiz 955-. doi: 10.1016/j.cgh.2008.03.021. Epub 2008 Jun 16.

16. Parra-Blanco A, Kaminaga N, Kojima T, Endo $\mathrm{Y}$, Uragami N, Okawa N, Hattori T, Takahashi H, Fujita R. Hemoclipping for postpolypectomy and postbiopsy colonic bleeding. Gastrointest Endosc. 2000:51(1):37-41.

17. Sorbi D, Norton I, Conio M, Balm R, Zinsmeister A, Gostout CJ. Postpolypectomy lower Gl bleeding: descriptive analysis. Gastrointest Endosc. 2000;51(6):690-6.

18. ASGE Standards of Practice Committee, Fisher DA, Maple JT, BenMenachem T, Cash BD, Decker GA, Early DS, Evans JA, Fanelli RD, Fukami N, Hwang JH, Jain R, Jue TL, Khan KM, Malpas PM, Sharaf RN, Shergill AK, Dominitz JA. Complications of colonoscopy. Gastrointest Endosc. 2011;74(4): 745-52. doi:10.1016/j.gie.2011.07.025

19. Nagata N, Niikura R, Aoki T, Moriyasu S, Sakurai T, Shimbo T, Shinozaki M, Sekine K, Okubo H, Watanabe K, Yokoi C, Yanase M, Akiyama J, Uemura N. Role of urgent contrast-enhanced multidetector computed tomography for acute lower gastrointestinal bleeding in patients undergoing early colonoscopy. J Gastroenterol. 2015;50(12):1162-72. doi:10.1007/s00535015-1069-9. Epub 2015 Mar 27.

20. Koh FH, Soong J, Lieske B, Cheong WK, Tan KK. Does the timing of an invasive mesenteric angiography following a positive $\mathrm{CT}$ mesenteric angiography make a difference? Int J Colorectal Dis. 2015;30(1):57-61. doi:10.1007/s00384-014-2055-z. Epub 2014 Nov 4.

21. Copland A, Munroe CA, Friedland S, Triadafilopoulos G. Integrating urgent multidetector $C T$ scanning in the diagnostic algorithm of active lower $\mathrm{Gl}$ bleeding. Gastrointest Endosc. 2010;72(2):402-5. doi:10.1016/j.gie.2010.04. 014. Review.

22. Sabharwal R, Vladica P, Chou R, Law WP. Helical CT in the diagnosis of acute lower gastrointestinal haemorrhage. Eur J Radiol. 2006;58(2):273-9. Epub 2006 Jan 18 